\title{
A comprehensive review on liposomes: A vesicular system for drug delivery
}

\author{
Vijay Kumar, Kapil Kumar*, Ikram, Aparna Joshi and Deepak Teotia \\ Department of Pharmaceutics, Global Institute of Pharmaceutical Education and Research, Kashipur-244713, Uttara hand, \\ India.
}

GSC Biological and Pharmaceutical Sciences, 2022, 18(02), 331-337

Publication history: Received on 19 January 2022; revised on 24 February 2022; accepted on 26 February 2022

Article DOI: https://doi.org/10.30574/gscbps.2022.18.2.0084

\begin{abstract}
In the last few years, many techniques have been utilized to enhance the pharmacological activity of any Active Pharmaceutical Drug [API] resulting in better bioavailability and lesser side effects. Liposomal drug delivery has evolved in this manner to increase the drug effect and reduce side effects. This review article focuses on all the aspects of liposomes with formulation methodology and characterization. Liposomes seem the first choice for designing a drug delivery system.
\end{abstract}

Keywords: Liposomes; Bioavailability; Liposomal drug delivery; API

\section{Introduction}

Many drugs experience very low bioavailability, poor pharmacokinetic profile, and high side effects which may be because of poor solubility of the drug or because of the physicochemical nature of the drug. To get better results and boost the therapeutic efficacy of the narrow range drugs nanotechnology has shown significant outcomes. Liposomes are one of the parts of the Nano-system with notable outcomes [1-4].

Liposomes are colloidal vesicles made up of one or more lipid bilayers surrounded by aqueous compartments. Liposomes encapsulate different types of drugs like antibiotics, antifungal, anticancer, proteins, hormones, peptides etc. Many drugs achieve therapeutically level for very less time because of metabolism of drug Liposomes can act as a promising carrier to delivery of drugs and to archives therapeutics level [5].

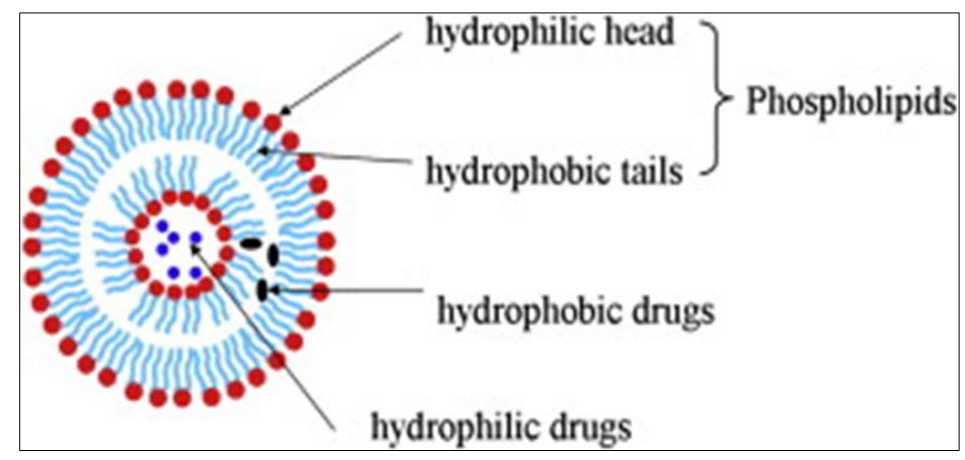

Figure 1 Liposome

\footnotetext{
*Corresponding author: Kapil Kumar

Department of Pharmaceutics, Global Institute of Pharmaceutical Education and Research, Kashipur- 244713, Uttarakhand, India. Copyright $(2022$ Author(s) retain the copyright of this article. This article is published under the terms of the Creative Commons Attribution Liscense 4.0.
} 


\subsection{Advantages of liposomes [6-9]}

- Liposomes act with the following benefits

- Liposomes can deliver a wide range of drug candidates (e.ghydrophilic, hydrophobic and amphipathic)

- Completely biodegradable, biocompatible, and non-toxic

- Reduces side effects especially in the case of chemotherapeutics agents wherein very low concentration drug show harmful effects.

- Minimizes contact of sensitive tissues to harmful drugs.

\subsection{Limitations of liposome}

- Manufacturing is very costly.

- Leaking and fusion of incorporated drug is a measure problem with liposomes.

\section{Types of liposomes [10-13]}

Liposomes are classified into different categories.

\subsection{Based on Vesicle size}

\subsubsection{Unilamellar vesicles}

- Small unilamellar vesicles (SUV) (Size- $40-80 \mathrm{~nm}$ )

- Medium unilamellar vesicles (MUV)(Size $-40-80 \mathrm{~nm}$ )

- Large unilamellar vesicles (LUV)(Size100 nm-1,000 nm)

\subsubsection{Oligolamellar vesicles (OLV)}

OLVs are made up of 10-20 lipid bilayers enclosed by internal volume.

\subsubsection{Multilamellar vesicles (MLV)}

They have many lipid bilayers. MLVs are prepared in a different manner. The structure is like layers of an onion. The central part is made up of LUV/MLV.

\subsection{Based on techniques of liposome preparation}

- REV: Reverse Phase Evaporation Method. This method is used to prepare Single or oligo lamellar and Multilamellar vesicles [14].

- SPLV: Stable Plurilamellar Vesicles

- FATMLV: Frozen and Thawed method used to prepare MLV.

- VET: Extrusion technique used to prepare different types of vesicles

- DRV: Dehydration-rehydration method is used to prepare different type of vesicles.

\subsection{Based upon composition and application}

- Conventional Liposomes (CL): Made up of neutral and negatively charged cholesterol and phospholipids ${ }^{15}$.

- Fusogenic Liposomes (RSVE)

- $\quad$ H sensitive Liposomes: Phospholipids such as DOPE with either

- Long Circulatory (Stealth) Liposomes (LCL): LCL contains PEG (polyethylene glycol) which is termed pegylation.Pegylation increases circulation of liposomes in the body by reducing its body clearance ${ }^{16}$.

\section{Methods of preparation of liposomes [17-22]}

Different methods are utilized to prepared liposomes.

\subsection{General method of liposomes preparation}

It involves following steps 
Drying of lipid from organic solvent followed by lipid dispersion in aqueous media followed by liposomes purification. At last analysis of liposomes.

\subsection{Passive Loading Techniques}

\subsubsection{Mechanical dispersion methods}

Lipid hydration method

Most common method of MLV formation.

This method involves drying of lipid solution to form a thin layer at the bottom of RBF. This film is further hydrated using aqueous buffer and overtaxing the mixture. The drug compound to be used to form liposomes is added either in that buffer or in organic solvent as per their solubility profile [2]3.

\subsubsection{Micro emulsification}

High pressure homogenizer is used for this method. This method is used to prepare SLVS. By using high shear stress lipid composition is micro emulsified.

\subsubsection{Dried reconstituted vesicles}

This method involves liposomes are mixed in an aqueous solution having drug or a mixture of lyophilized protein which is further dehydrated.

\subsubsection{Freeze thaw method}

Results in formation of SUVs using slow thawing method [24].

\subsubsection{Solvent Dispersion}

Ethanol injection

A lipid solution of ethanol is mixed into aqueous buffer resulting formation of MLVs.

Ether infusion

A lipid solution is added in diethyl ether and injected slowly to a solution of drug compound to be encapsulated.

Detergent

Detergents can solubilize lipids at CMC. After removal of detergents micelles becomes rich in phospholipids and join together to form LUVs.

\subsection{Active Loading Techniques}

\subsubsection{Proliposomes}

In this method drug molecules and lipid both are coated with soluble carrier to form freely flowing granules in proliposomes. When hydrated results in an isotonic liposomal suspension [25].

\subsubsection{Lyophillization}

Lyophilization is the process of water removal under reduced pressure in a frozen state. This method is utilized for drying of thermolabile products.

\section{Evaluation parameters of liposomes}

- Particle Size Determination

- Surface Charges

- Encapsulation Efficiency (EE)

- Phase Behavior

- Drug Release Study 


\subsection{Particle Size Determination}

Both particle size and particle size distribution of liposomes can be determined by using following methods;

- $\quad$ Laser light scattering

- Transmission electron microscopy

\subsection{Surface charges}

The method involved in the measurement of surface charge is based on free-flow electrophoresis of Multi-Layer Varistors (MLVs).

- It utilizes a cellulose acetate plate dipped in sodium borate buffer of pH 8.8

- $\quad$ About $5 \mathrm{~N}$ moles of lipid samples are applied on to the plate, which is then subjected to electrophoresis for 30 minutes.

- $\quad$ The liposomes gel gets bifurcated depending on their surface charges.

This technique can be used for determining the heterogeneity of charges in the liposomes suspensions as well as to detect any impurities such as fatty acids [26].

\subsection{Encapsulation Efficiency (EE)}

The quantity of drug entrapped in the liposomes helps to estimate the behavior of the drug in a biological system. The $\%$ of drug encapsulation is done by first separating the free drug fraction from encapsulated drug fraction. The encapsulated fraction is then made to leak off the liposomes into an aqueous solution using suitable detergents [27].

\subsection{Phase Behavior}

The phase behavior of liposomes can be determined by using differential scanning calorimetry (DSC). Around 2 mg sample is taken out and placed in a sealed aluminum pot. And an empty aluminum pot is used as a reference. The impurities determination is performed by using a heating rate of $10^{\circ} \mathrm{C} / \mathrm{min}$. DSC equipment should be $1^{\circ}$ calibrated.

\subsection{Drug Release Study}

$500 \mathrm{ml} \mathrm{20 \%}$ ethanol was used as the release medium; $10 \mathrm{ml}$ release medium was drawn and placed into a dialysis bag The dialysis bag was then clamped and attached on the paddle of a dissolution apparatus; 5 ml drug-containing liposomes and $5 \mathrm{ml}$ ethanol solution of drug with the same drug content as the liposomes, were respectively dissolved in the dialysis vessel, with stirring at $37^{\circ} \mathrm{C}$ and $300 \times \mathrm{g}$. A sampling of $100 \mu \mathrm{l}$ liquid from the dialysis bag was conducted at $1,2,4,6,8,10,12$ and $24 \mathrm{~h}$ for sample determination and calculation of the accumulative release rate. A release curve was drawn using the time $(\mathrm{t})[28]$.

\section{Marketed formulations of liposomes}

In 1995, Doxil (PEGylated liposome-encapsulated doxorubicin) was the first liposomal formulation approved by the US FDA. There are some liposomal formulations available in market for human use (table1).

\subsection{Applications of Liposomes [9-13]}

Liposomes already established a wide are of applications. Some of them are discussed here as follows.

\subsubsection{Respiratory Disorders}

Liposomes found very beneficial in respiratory disorders with sustain release, better stability of drug product with minimum side effects in comparison of convention aerosols. Liquid and dry both forms can be taken in liposomal form with nebulization.

\subsubsection{Tumor therapy- Carrier}

Liposomes have already been established as nanocarrier in chemotherapy treatment. Many drug formulations have already been approved for chemotherapy. 


\subsubsection{Immunological adjuvants in vaccines}

The liposomal formulation are used as immunoadjuvant and in immunodiagnostic.

\subsubsection{Ophthalmic Disorders}

Liposomes have shown positive results against many eye disorders, including dryness of eyes, corneal transplant rejection etc. Drug verteporfin has been approved as a liposomal formulation for eye disorders.

\subsubsection{Pulmonary Application}

Liposomes are one of the best tools for pulmonary delivery of drugs because of their better solubility profile.

\subsubsection{Liposomes in Cosmetics-}

Liposomes are also used in the cosmetic industry because of their release pattern and similarity to the cell membrane.

\subsubsection{Site specific targeting}

The immunoliposomes are capable to identify and binding to target cells with higher affinity.

5.1.8. Liposomes as protein drug delivery-

Liposomes are utilized in increase drug solubilization.

\subsubsection{Gene therapy}

Liposomes a reutilized widely in gene applications to cure diseases.

Table 1 Market Formulations of liposomes

\begin{tabular}{|l|l|l|l|}
\hline S. No & Product Name & Drug Name & Manufacturer \\
\hline 1 & Ambisome & Amphotericin B & Nexstar Pharmaceuticals Inc.CO \\
\hline 2 & Abelcet & Amphotericin B & The Liposome company N.J. \\
\hline 3 & Amphocil & Amphotericin B & Sequus Pharmaceuticals Inc.CA \\
\hline 4 & Doxil & Doxorubicin & Sequus Pharmaceuticals Inc. \\
\hline 5 & Daunoxome & Daunorubicin & Nexstar Pharmaceuticals Inc.CO \\
\hline 6 & Mikasome & Amikacin & Nexstar Pharmaceuticals Inc.CO \\
\hline 7 & DC99 & Daxorubicin & The Liposome company N.J. \\
\hline 8 & Epaxel & Hepatitis A vaccine & Swiss Serum institute Switzerland \\
\hline 9 & ELA max & Lidocaine & Biozone Labs, Ca, USA \\
\hline
\end{tabular}

\section{Advancements in liposomes}

\subsection{Ethosomes}

They are well-organized to deliver to the skin made up of soya phosphatidylcholine and $30 \%$ ethanol.

\subsection{Immuno liposomes}

They were improved with antibodies.

\subsection{Niosomes}

Small unilamellar vesicles made from non- ionic surfactants. 


\subsection{Stealth liposomes}

Stealth liposomes possess modified and long half-life in circulation. PEG coating is used to prepare these liposomes.

\section{Conclusion}

Tradition pharmaceutical doses forms have many limitations which can be overcome by use of a liposomal drug delivery system. Nano carriers systems are developed as a potential system in the delivery of drugs. Liposomes act as a Nano carriers system that can act and reach to a specific site, tissue, or organ. As mentioned in the above discussion many anti-cancerous drugs are now available in liposomal form. To get better results from liposomal formulation it should be designed properly resulting in better bioavailability of and lesser side effects. Liposomes are one of the best choices of Nano carrier in drug delivery, site-specific drug delivery, specific organ, and receptor targeting.

\section{Compliance with ethical standards}

\section{Acknowledgments}

Authors are thankful for the management of the institute to provide necessary facilities for this work.

\section{Disclosure of conflict of interest}

No conflict of interest is associated.

\section{References}

[1] De Marie S, Janknegt R, Bakker-Woudenberg IAJM. Clinical use of liposomal and lipid-complexed amphotericin B. J. Antimicrob. Chemother.1994;33: 907-916.

[2] Obanewa OA, Oyeniran OT. Development and estimation of anti-inflammatory activity of topical etoricoxibemulgel by carrageenan induced paw oedema method. Universal Journal of Pharmaceutical Research. 2019; 4(3): 22-26.

[3] Mishra H, Chauhan V, Kumar K, Teotia D, A comprehensive review on Liposomes: a novel drug delivery system, Journal of Drug Delivery and Therapeutics. 2018; 8(6):400-404

[4] Dingwoke John Emeka Francis, Felix Sunday Yusuf. Development and evaluation of nanosponges loaded extended release tablets of lansoprazole. Universal Journal of Pharmaceutical Research. 2019; 4(1): 24-28.

[5] Emanuel N, Kedar E, Bolotin EM, Smorodinsky NI,Barenholz Y. Preparation and characterization ofdoxorubicinloaded sterically stabilized immunoliposomes.Pharm. Res. 1996; 13: 352-35.

[6] Nweje-Anyalowu Paul C, Anyalogbu Ernest AA, White Alalibo Jim. Design and evaluation of chronotherapeutic pulsatile drug delivery system of Cilnidipine. Universal Journal of Pharmaceutical Research. 2017; 2(5): 15-18.

[7] Felgner JH, Kumar R, Sridhar CN, Wheeler CJ, Tsai YJ, Border R, Ramsey P, Martin M, Feigner PL. Enhanced gene delivery and mechanism studies witha novel series of cationic lipid formulations. J. Biol. Chem.1994; 269: 25502561.

[8] Peter OI, Ifeoma UC. Development and evaluation of Albendazole microcapsule for colonic drug delivery system. Universal Journal of Pharmaceutical Research. 2017; 2(2): 4-7.

[9] HAH Rongen, ABult, WP van, Bennekom J Immuno. Methods. 1997; 204:105-133.

[10] Kaur G, Paliwal S. Formulation and evaluation of etoricoxib microbeads for sustained drug delivery. Universal Journal of Pharmaceutical Research. 2019; 4(1): 35-39.

[11] New RRC. Preparation of liposomes. In: New RRC.(Ed.), Lipsomes: a practical approach, IRL Press, Oxford. 1990; 33: 104.

[12] Sunday OS. Colon-targeted drug delivery systems: design, trends and approaches. Universal Journal of Pharmaceutical Research. 2017; 2(4): 46-50.

[13] Jr F Szoka, DPapahadjopoulos. Proc. Natl. Acad. Sci. USA. 1978; 60:4194-4198. 
[14] Elsaied EH, Dawaba HM, Ibrahim EA, Afouna MI. Investigation of proniosomes gel as a promising carrier for transdermal delivery of Glimepiride. Universal Journal of Pharmaceutical Research. 2016; 1(2): 1-10.

[15] Zaquiyya Naaz*, Dr. Kapil Kumar and Deepak Teotia. A Comprehensive Review on Liposomes. International Journal of Modern Pharmaceutical Research. 2020, 4(5), 70-74.

[16] Ugochukwu AE, Nnedimkpa OJ, Rita NO. Preparation and characterization of Tolterodine tartrate proniosomes, Universal Journal of Pharmaceutical Research. 2017; 2(2): 1-3.

[17] R Bajoria, SF Contractor. Effectofthe Size of Liposomes on the Transfer andUp- take of Carboxyfluorescein by the Perfused Human Term Placenta, J. Pharm. Pharma- col.1997; 49:675-681.

[18] Anwar W, Dawaba HM, Afouna MI, Samy AM.Screening study forformulation variables in preparation and characterization of candesartan cilexetil loaded nanostructured lipid carriers. Universal Journal of Pharmaceutical Research. 2019; 4(6):8-19.

[19] Vishvakrama P, Sharma S. Liposomes: anoverview. Journal of Drug Delivery and Therapeutics. 2014; 47-55.

[20] Chioma ED. Formulation and evaluation of etodolacniosomes by modified ether injection technique. Universal Journal of Pharmaceutical Research. 2016; 1(1): 1-4.

[21] Samadikhah HR, Majidi A, Nikkhah M, Hosseinkhani S. Preparation, characterization, and efficient transfection of cationic liposomes and nanomagnetic cationic liposomes. Int J Nanomedicine. 2011; 6: 2275-2283.

[22] Mathur P, Mathur CK, Mathur K. Oral drug delivery of insulin in diabetes mellitus: an attractive alternate to overcome invasive route. Universal Journal of Pharmaceutical Research. 2018; 3(6): 45-48.

[23] Gurleen Kaur, Zaquiyya Naaz, Kapil Kumar, Deepak Teotia. Formulation and Evaluation of Aceclofenac Liposomes, Journal of Drug Delivery and Therapeutics. 2021; 11(5):71-79.

[24] Elsaied EH, Dawaba HM, Ibrahim ESA, Afouna MI. Effect of pegylated edge activator on Span 60 based nanovesicles: comparison between Myrj 52 and Myrj 59. Universal Journal of Pharmaceutical Research. 2019;4(4):1-8.

[25] Li X, Chen D, Le C, Zhu C, Gan Y, Hovgaard L. Novel mucus- penetrating liposomes as a potential oral drug delivery system: preparation, in vitro characterization, and enhanced cellular uptake. Int J Nanomedicine. 2011; 6:31513162.

[26] John DF, Yunus AA, Chigbo UJ, Paul US, Ikenna E. Tolnaftate loaded liposomes-design, and in-vitro evaluation. Universal Journal of Pharmaceutical Research. 2016; 1(2): 29-31.

[27] Ejiogu Deborah Chioma. Formulation and evaluation of etodolacniosomes by modified ether injection technique. Universal Journal of Pharmaceutical Research. 2016; 1(1): 1- 6.

[28] Nwobodo NN, Adamude FA, Dingwoke EJ, Ubhenin A. Formulation and evaluation of elastic liposomes of decitabine prepared by rotary evaporation method. Universal Journal of Pharmaceutical Research. 2019; 4(3): $1-5$. 